\title{
Experimental evaluation of a vibro-impact model for two adjacent shear-building structures
}

\author{
Marcus Varanis ${ }^{1, *}$, Arthur Mereles ${ }^{1}$, Anderson Silva ${ }^{1}$, José Balthazar $^{2}$, Ângelo Tusset ${ }^{2}$, and \\ Clivaldo Oliveira ${ }^{1}$, \\ ${ }^{1}$ Federal University of Grande Dourados, Dourados, Brazil \\ ${ }^{2}$ Federal Technological University of Paraná, Paraná, Brazil.
}

\begin{abstract}
The vibro-impact phenomenon is found in many engineering applications, from impact of floating ice with ships to rubbing between the stator structure and rotor blades in turbomachinery, and in most cases it is important to know the implication of this phenomenon in the mechanical system. This is often done by proposing vibro-impact models for describing the behavior of the system when subjected to periodically impacts. However, this modelling may be challenging due to the strongly nonlinear characteristics of the impact phenomenon. Therefore, this paper presents a vibro-impact model of two shearbuilding structures positioned side by side, where one of them is driven by an unbalanced DC motor. The structures were modeled using the Euler-Bernoulli beam theory and the contact was modeled based on the Hertz contact theory. In order to validate the model their responses were compared with experimental signals.
\end{abstract}

\section{Introduction}

In most cases, the motion of vibro-impact systems are very complex due to the non-smooth characteristic resulting from impact [1]. This characteristic arises from the sudden change in the velocity of the system after the contact. Thus, vibro-impact systems are commonly modeled using non-smooth differential equations. The firsts models of vibro-impact systems were based on the hypothesis of hard impact, which considers the bodies to be rigid and the impact to occur instantaneously. The parameter of interest in this approach is the coefficient of restitution (COR), which is a measure of the energy loss in the impact. Some examples of studies considering hard impacts are presented in [2-5]. This approach, despite being simple and easy implemented in some cases, is unable to model systems with large contact time, as well as impact with deformed bodies.

Another approach used in the modeling of vibro-impacts systems consists in introducing a function to model the deformation of the impacting bodies during their contact. These are called soft impact models, and they model the phenomenon with more accuracy than the models based on hard impacts, since in the former the contact force takes a finite value and the contact time are not instantaneously. Some works that treated soft impact models

\footnotetext{
*e-mail: marcusvaranis@ufgd.edu.br
} 
are [6-9]. The functions introduced are generally force-deformations or/and force-velocity relations and can be linear or nonlinear. An important function used to represent soft impacts is obtained through the Hertz contact theory. Some applications of the Hertz force law in vibro-impact systems are presented in [10-15].

In this paper, the dynamics of a mechanical system consisting of two shear-building structures subjected to impacts is studied. The structures were modeled as a continuous system using the Euler-Bernoulli beam theory and the contact was modeled using the Hertz contact theory. The shear-building structures are positioned side by side, being one of them driven by an unbalanced DC motor. In order to evaluate the model proposed, their responses were compared with signals obtained from an experimental procedure.

\section{Vibro-impact model}

The vibration model consist of two beams clamped at one end and with tip masses at the other separated by a distance $d$, as depicted in Figure 1. The beams have the same length denoted by $L$, which is the point considered for the impact to happen. On top of one of the beams there is an unbalance DC motor with an unbalance mass $m_{u}$ and a distance to the rotating axis of $e$. The displacements of the structures are given by $w(x, t)$ and $v(x, t)$. The equations of motion for the beams according to the Euler-Bernoulli beam theory are given by,

$$
\begin{gathered}
E_{1} I_{1} \frac{\partial^{4} w}{\partial x^{4}}(x, t)+\rho_{1} A_{1} \frac{\partial^{2} w}{\partial t^{2}}(x, t)+c_{1} \frac{\partial w}{\partial t}(x, t)=\delta_{d}(x-L) m_{u} e \omega^{2} \cos (\omega t)-\delta_{d}(x-L) F_{c}(t) \\
E_{2} I_{2} \frac{\partial^{4} v}{\partial x^{4}}(x, t)+\rho_{2} A_{2} \frac{\partial^{2} v}{\partial t^{2}}(x, t)+c_{2} \frac{\partial v}{\partial t}(x, t)=\delta_{d}(x-L) F_{c}(t)
\end{gathered}
$$

where the subscripts ${ }_{1,2}$ refer to each the beams, $E$ is the Young's modulus, $I$ is the area moment of inertia, $\rho$ is the density, $A$ is the cross-section area, $c$ is the viscoelastic damping coefficient, $\delta_{d}$ is the Dirac delta function, $\omega$ is the excitation frequency or the motor rotating speed, $L$ is the length of the beams and $F_{c}$ is the contact force. The contact force considered in this work is based on the Hertz contact theory and is given by [10],

$$
F_{c}(t)=k_{h} \delta(t)^{3 / 2}\left(1+c_{h} \dot{\delta}(t)\right)
$$

where $\delta$ is the indentation or relative penetration, $k_{h}$ is the impact stiffness which depends on the bodies mechanical properties, $c_{h}$ is the impact damping coefficient which depends on the

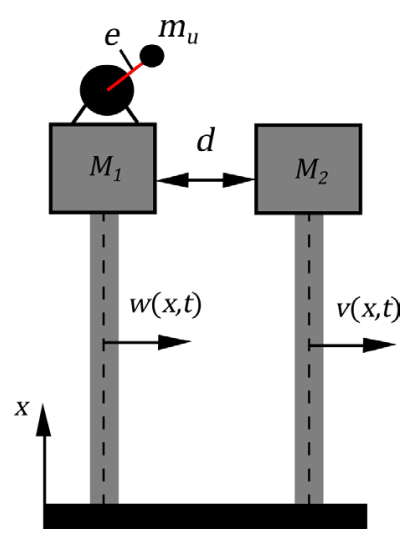

Figure 1: Model of the mechanical system studied. 
energy loss after the contact, and the dot represent a time differentiation. The indentation for this model will be, $\delta=v(L, t)-w(L, t)-d$. Therefore, one can write the equation for the contact force as,

$$
F_{c}(t)=k_{h}[v(L, t)-w(L, t)-d]^{3 / 2}\left[1+c_{h}\left(\frac{\partial v}{\partial t}(L, t)-\frac{\partial w}{\partial t}(L, t)\right)\right]
$$

The contact force has always a positive value, thus when $F_{c}<0$ or $\delta<0$ it is assumed that $F_{c}=0$, which means that the beams are not in contact. To obtain the responses of the beams, the modal expansion method was used, which considers the displacement of the beams to be the multiplication of two functions of only $x$ and $t$, i.e.,

$$
\begin{aligned}
w(x, t) & =\sum_{n=1}^{\infty} \phi_{n}(x) W_{n}(t) \\
v(x, t) & =\sum_{n=1}^{\infty} \psi_{n}(x) V_{n}(t)
\end{aligned}
$$

where $\phi_{n}(x)$ and $\psi_{n}(x)$ are the mass normalized eigenfunctions of the two beams, and $W_{n}(t)$ and $V_{n}(t)$ are the modal coordinates of the beams for the nth mode. In order to obtain the responses, the equations of motions where solved using the Runge-Kutta method. In the simulations the number of modes was considered was $N=3$.

\section{Experimental setup}

The experimental setup consist in two identical single-story shear-building structures positioned side by side by a distance of $1 \mathrm{~mm}$, being one of the structures excited by an unbalanced DC motor as shown in Figure 2. The columns of the shear-building structures were manufactured using ASTM A-36 steel with dimensions $1.75 \mathrm{~mm} \times 76.2 \mathrm{~mm} \times 300 \mathrm{~mm}$, which correspond to the thickness, width and length, respectively. The floor and the top of the structures are made from polypropylene plates with dimensions of $15 \mathrm{~mm} \times 76.2 \mathrm{~mm} \times 400 \mathrm{~mm}$. The DC motor used was a Mabuchi DC motor model C2162-60006 powered by a power source of Minipa model MPL-3303M, and it was mounted in the middle of the top of the excited structure as shown in Figure 2. The motor was unbalanced by placing a mass of 6.59 $\mathrm{g}$ in a distance of $15 \mathrm{~mm}$ from the axis of rotation. In addition, the structures were bolted in an inertial bench to prevent vibrations from other sources to interfere in the measurements.

In order to measure the accelerations of the structures, two accelerometers were positioned at the top of the no impacting column of each structure. The accelerometers used were from Dytran Instruments Inc. model 2335F1, which have sensitivity of $9.89 \mathrm{mV} / \mathrm{g}$. For the acquisition and data analysis a LMS Scadas Mobile model VB8-II from Siemens was used.

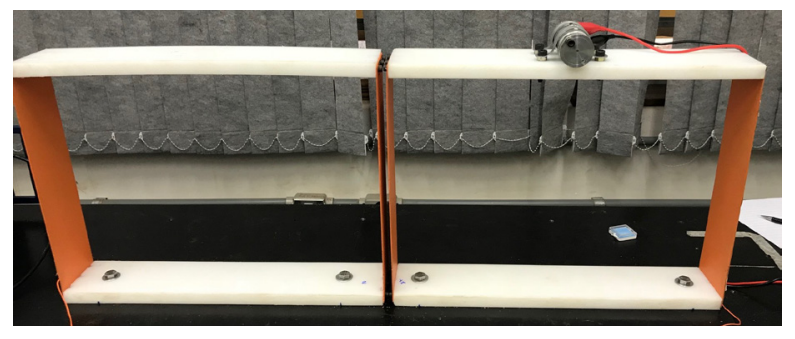

Figure 2: Mechanical system studied: two identical adjacent shear-building structures. 


\section{Results and discussion}

The experiments were carried out at the Intelligent Materials and Control Laboratory in the Ilha Solteira Campus of the São Paulo State University (UNESP). The signals were obtained using a sample frequency of $2048 \mathrm{~Hz}$ and a time of measurement of $14 \mathrm{~s}$. The system was analyzed in three different rotational speeds of the unbalanced motor, which corresponded to the regions of resonance, pre-resonance and post-resonance. The resonance frequency is related to the first natural frequency of structure 1. The gap distance and the time step used in the simulations were $d=1 \mathrm{~mm}$ and $\Delta t=6.67 \times 10^{-6} \mathrm{~s}$, respectively. The impact stiffness and damping were $k_{h}=2.307 \times 10^{10} \mathrm{~N} / \mathrm{m}^{3 / 2}$ and $c_{h}=4.378(\mathrm{~m} / \mathrm{s})^{-1}$, and the damping factor $\zeta=0.003$, which was the same for both structures.

Figure 3 presents the case when the excitation frequency matched the first natural frequency of Structure 1, where the peaks represent the accelerations due to contact. The blue graphs correspond to the accelerations of structure 1, while the red ones correspond to structure 2 accelerations. As it can be expected, this was the case with the most intense contact. By comparing the numeric signals, Figures $3 \mathrm{a}$ and $3 \mathrm{c}$, with their corresponding experimental ones, Figures $3 b$ and $3 d$, it is noted that the model presented responses very similar to the ones seen in the experiment. Also, the peak accelerations values of the numeric signal were very close to the experimental ones, which shows that the impact stiffness and damping were suitable for the vibro-impact system. In addition, it is worth noting that only the first mode of vibration of structure 2 was excited by the impacts, since the amplitudes of the higher modes of structure 1 were unable to surpass the gap distance in the simulations.

In the other case studied, presented in Figure 4, the excitation frequency was slightly higher than the natural frequency, specifically $5 \%$ higher, so that the beating phenomenon could be seen. In this case the numeric signals predicted peak accelerations much higher than the measured values, as one can note comparing Figures $4 \mathrm{a}$ and $4 \mathrm{c}$ with Figures $4 \mathrm{~b}$ and $4 \mathrm{~d}$. Also, the number of impacts per period of oscillation was not the same for the numeric and experimental cases, since in the latter more impacts are seen in the vibration of the system.
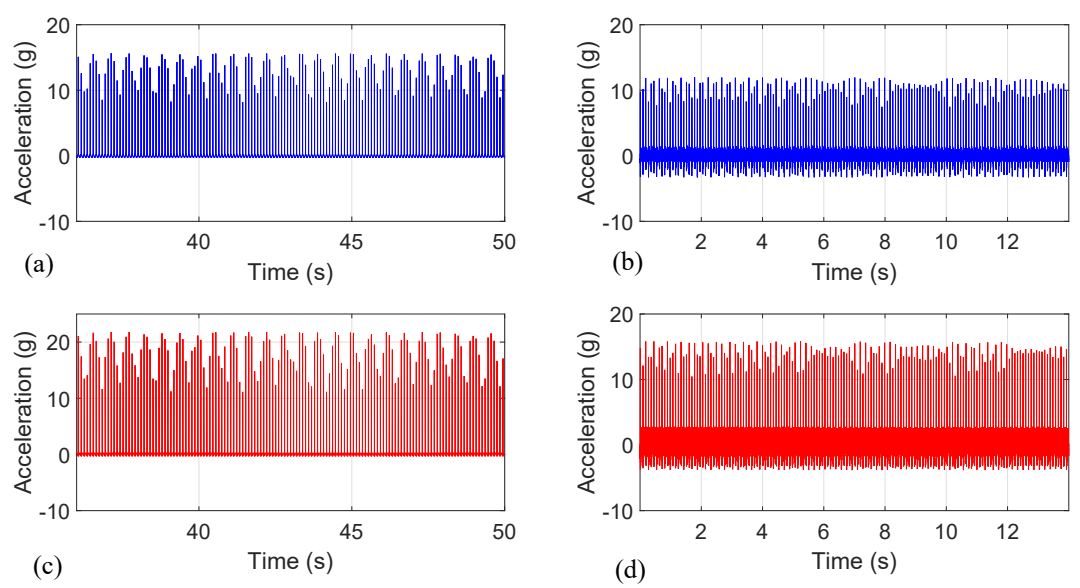

Figure 3: Comparison between the experimental and the numeric signals for the excitation frequency at the resonance: (a) and (b) Structure 1 numeric and experimental accelerations, (c) and (d) Structure 2 numeric and experimental accelerations. 

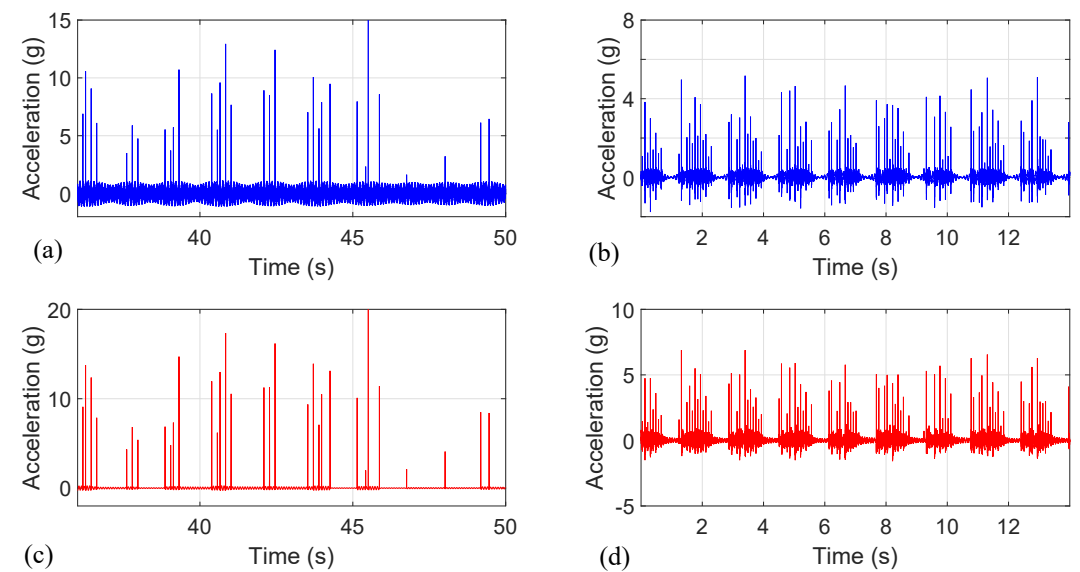

Figure 4: Comparison between the experimental and the numeric signals for the excitation frequency at the post-resonance: (a) and (b) Structure 1 numeric and experimental accelerations, (c) and (d) Structure 2 numeric and experimental accelerations.
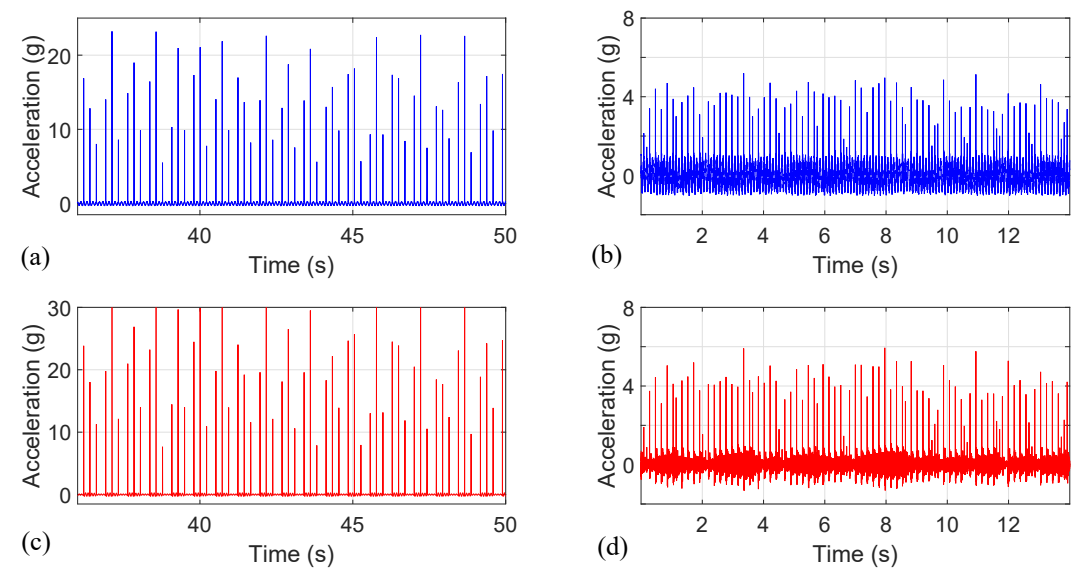

Figure 5: Comparison between the experimental and the numeric signals for the excitation frequency at the pre-resonance: (a) and (b) Structure 1 numeric and experimental accelerations, (c) and (d) Structure 2 numeric and experimental accelerations.

In the case where the system was in the region of pre-resonance, presented in Figure 5, the excitation frequency was $95 \%$ the first natural frequency of structure 1 . It can be seen by comparing the numeric and the experimental signals, Figures $5 \mathrm{a}$ and $5 \mathrm{c}$ with Figures $5 \mathrm{~b}$ and $5 \mathrm{~d}$, the peak acceleration values were very different for this case. However, the impacts per period of oscillation of the numeric signal were very close to the experimental ones, which can be noted analyzing the peaks of acceleration in the figures. Thus, despite predicting higher values of acceleration, the numeric signals were suitable with the experimental ones regarding the dynamic behavior of the system. 


\section{Conclusions}

This paper presented a continuous vibration model for two shear-building structures subjected to impacts, being one of the structures driven by an unbalance DC motor. The impacts were modeled by means of the Hertz contact theory, which states a nonlinear relation between the contact force and the indentation of the bodies. In order to validate the model, an experimental procedure was proposed, where the accelerations of the structures were measured using accelerometers. Three cases were analyzed: in the resonance region, in the post-resonance region, in the pre-resonance and a free vibration case. In the resonance case, the model predicted values for the peak accelerations which were very close to the experimental data. The dynamic behavior, impacts per period of oscillation, was also in according to the experiments, showing that the model gives also a good qualitative idea of the mechanical system. However, in the cases of post- and pre-resonance, the values predicted by the model were much higher than the measured ones, although in the latter the dynamic behavior was close to the experiments.

\section{References}

[1] R.A. Ibrahim, Vibro-impact dynamics: modeling, mapping and applications, Vol. 43 (Springer Science \& Business Media, 2009)

[2] D. Wagg, S. Bishop, Journal of Sound and vibration 236, 176 (2000)

[3] F. Peterka, Prague: Academia (1981)

[4] F. Pfeiffer, On unsteady dynamics in machines with plays, in Proc. 7th World Congress on TMM (1987), Vol. 1, pp. 471-423

[5] M. Papadrakakis, H. Mouzakis, N. Plevris, S. Bitzarakis, Earthquake engineering \& structural dynamics 20, 981 (1991)

[6] S.W. Shaw, P. Holmes, Journal of Sound and Vibration 90, 129 (1983)

[7] S. Aoki, T. Watanabe, Stationary Random Vibration of Mechanical System With Collision Characteristics, in Vibration Conference (1997), Vol. 1, pp. 185-190

[8] M. Wiercigroch, V. Sin, Journal of Applied Mechanics 65, 657 (1998)

[9] F. Peterka, A. Tondl, Chaos, Solitons \& Fractals 19, 1283 (2004)

[10] L. Půst, F. Peterka, Meccanica 38, 99 (2003)

[11] S. Muthukumar, R. DesRoches, Earthquake engineering \& structural dynamics 35, 811 (2006)

[12] L. Pust, Models of Weak Stops-Application to 2DOF System, in Proc. 10th World Congress on TMM (1999), Vol. 4, pp. 20-24

[13] W. Serweta, A. Okolewski, B. Blazejczyk-Okolewska, K. Czolczynski, T. Kapitaniak, International Journal of Mechanical Sciences 89, 194 (2014)

[14] H.A. Navarro, J.M. Balthazar, R.M. Brasil, Vibrations due to Impact in a Non Ideal Mechanical System With a Non-Linear Hertzian Contact Model, in ASME 2014 International Design Engineering Technical Conferences and Computers and Information in Engineering Conference (American Society of Mechanical Engineers, 2014), pp. V008T11A041-V008T11A041

[15] A. Mereles, M. Varanis, J.M. Balthazar, R. Rocha, C. Oliveira (2017) 